\title{
Performance Assessment of Channel Capacity of Co-operative Communication based System over Dual-hop Asymmetric RF/FSO system
}

\author{
Shyam Baboo Bambiwal, Rajveer Singh Yaduvanshi, Vijay Kumar Pandey
}

\begin{abstract}
Cooperative Communication Systems and Relay Selection approaches are recent techniques of wireless communication system that enhance the quality of service (QoS). This research paper probes the execution of the Radio-Frequency and Free Space Optical (RF/FSO) Co-operative system using network of relays. The Co-operative relay system is typically composed of node of a source, the nodes of relays and node of a destination. The source and relay link (S-R link) follow the Generalized-K Fading distribution channel. The link connecting the relay node/device and the destination ( $R-D$ link) follows $k-\mu$ Fading distribution channel. The relay is considered to adopt according to DF i.e Decode and Forward method of relaying and cooperatively assists in transmission to the destination node from the source node. The channel capacity is modelled using PDF (Probability Density Function) for the $\kappa-\mu$ distribution channel of fading for evaluation of the Dual-hop Asymmetric RF-FSO system. The performance and the analytical expressions of the new system proposed i.e. RF/FSO system were verified and validated using Monte Carlo simulation method.
\end{abstract}

Keywords: Co-operative communication system, Decode-and-Forward relaying, Channel capacity, Generalized-K, $\kappa-\mu, R F / F S O$ system.

\section{INTRODUCTION}

In recent years wireless communications have seen great advancement with technological development. Newer types of networks are being introduced to enhance communication capabilities. Cooperative Communication System is one such newly developed approach based upon broadcast nature of the wireless channel, with same advantage as of MIMO systems. In Cooperative Communication System, active nodes participating in communication, helps to execute the process of communication in distributed fashion [1]. One prime challenging task is to communicate through a wireless

Revised Manuscript Received on March 16, 2020.

* Correspondence Author

Shyam Baboo Bambiwal*, Research Scholar, Dr. A.P.J. Abdul Kalam Technical University, Sec-11, Jankipuram, Vistaryojna, Lucknow (U.P) India. Email: Sbambiwal@Gmail.Com

Rajveer Singh Yaduvanshi, Professor, ECE Department, Ambedkar Institute Of Advanced Communication Technologies And Research, Govt., Geeta Colony, New Delhi, India. Email: Yaduvanshirs007@Gmail.Com

Vijay Kumar Pandey, Professor \& Dean (2nd Shift) Department Of ECE, Noida Institute Of Engineering And Technology, Knowledge Park-II, Greater Noida, (U.P), India. Email: Vijaygpandey@Yahoo.Com

(C) The Authors. Published by Blue Eyes Intelligence Engineering and Sciences Publication (BEIESP). This is an open access article under the CC BY-NC-ND license (http://creativecommons.org/licenses/by-nc-nd/4.0/) channel, because the medium, in the first place, introduces substantial loss to the signal that is being transmitted. To overcome this, newer communication systems such as Optical Wireless Communication or FSO systems have developed. Main advantages of these newer systems are enhanced capacity and bandwidth when compared to traditional RF based communication systems.

A cooperative network relay system has an "S" as the source, a "D" as the Destination and " $\mathrm{N}$ " as the number of relays that operates in flat fading channels. Throughout the cooperative communication system, relay channels establish independent routes between the source (S) and the destination (D) stations. Each wireless user station in co-operative relay communication network is responsible for transmitting data and to participate in communication process as co-operative relay agent [2]. Cooperative communication protocols can be divided into two categories: One as Adaptive Relaying Schemes and other as Fixed Relaying Schemes. In the Fixed relaying protocol, using Amplify and Forward (AF) method which transmits an amplified input version to the "D" destination node. Also in the fixed relaying method using Decode and Forward (DF) relaying protocol, it decodes the received signals first, and then re-encodes them before they are transmitted to the " $\mathrm{D}$ " destination node. Any of these two i.e. AF or DF relaying protocol is chosen for the source ' $\mathrm{S}$ ' node and the destination ' $\mathrm{D}$ ' node to establish communication. By using its neighbouring devices as relays, cooperative communication system provides a reliable network form single antenna devices [3]-[6].

In this research paper, mathematical modelling is employed for channel capacity. Performance for of $\kappa-\mu$ Fading channel in the FSO link of cooperative relay network is evaluated, under DF relaying. The objectives of our work are as under,

- Development of a mathematical modelling for the proposed RF/FSO system and estimate channel capacity.

- To test the arithmetical results obtained and the performance of the system proposed using Monte Carlo simulations.

The remainder of this research paper is organized as follows: Section-II involves review of the literature. Section-III discusses/describe the proposed system, its research methodology and mathematical modelling. Section-IV describes its results and discussion for the proposed system. At last the Section V states our conclusion and scope for future work..

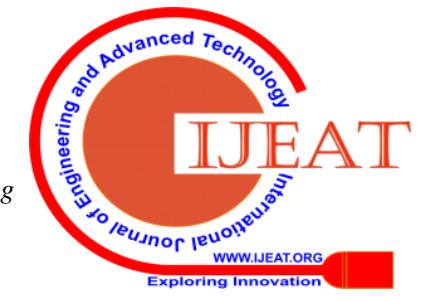




\section{Performance Assessment of Channel Capacity of Co-operative Communication based System over Dual-hop Asymmetric RF/FSO system}

\section{LITERATURE REVIEW}

Palliyembil V (2018) [7] has proposed numerical expressions for outage probability along with channel capacity approach for performance improvement of an RF/FSO system, in which, the source node to relay hop communication noticed Nakagami-m fading whereas the relay node-destination hop noticed M-distribution fading. The alternative useful technique for improving the communication performance of FSO is by making use of multiple transmitting and receiving apertures as well.

Performance Evaluation and comparison between AF and DF relaying schemes for Outrage capacity was done by Chen, $X$ et al. (2015)[8]. The explicit expressions in terms of transmit SNRs were derived. It is found that both methods show the same performance at high source transmission power.

Nauryzbayev, G et al. (2018)[9] examined the various wireless powered Dual-hop AF relaying protocols for Ergodic capacity and Outage probability over $\alpha-\mu$ Fading distribution Channel. The authors proposed analytical expressions in closed-form with respect to the H-functions, and Monte Carlo simulations have confirmed the results.

M. K. Simon \& M.-S. Alouini (2005)[10] acquire the outage probability of $\kappa-\mu$ Fading distribution Channels along with the generalized Marcum Q function. It is assumed the value of $(m-\mu)$ must not be a positive integer. However, the rest assumed parameters can take any arbitrary value in the fading model of the $\kappa-\mu$ distribution channel.

Tang, X et al. (2014)[11] carry out an investigation of the performance for multi-hop carried out in RF/FSO systems. Performance for End-to-End Serial transmission (or multi-hop) for optical wireless links have been dealt with previously by several researchers.

The pdf expression for Generalized $-\kappa$ fading channel was derived by Laourine, A et al. (2008)[12]. Results are calculated for expression of the channel capacity by adapting the optimum rate with adaptive power, optimum rate with constant power and channel inversion at a fixed rate. On the other hand, adapting Generalized- $\kappa$ for FSO link is not viable to take recourse to the relaying protocol.

The $\kappa-\mu$ fading model takes into account a signal which comprises multi-path wave clusters that propagate in a non-homogenous medium. The wave phases that are scattered are assorted randomly in the cluster [13]. $\eta-\mu$ fading distribution model is better suited for non Line of Site propagation (Non-LOS) conditions, which also includes single-sided Gaussian, Nakagami-q (Hoyt), Nakagami-m and Rayleigh fading models as unique case. While on the other hand, for Line of Site(LOS) signal propagation conditions, which included Nakagamin (Rice), RayleighNakagami-m, and One-Sided Gaussian fading models, the performance of $\kappa-\mu$ fading distribution model works well. D. B. da Costa et al., N. Y. Ermolova, K. Peppas et al. and D. Dixit et al. (2008-2012) Studies the execution of different points-to-points cooperative communication systems over the fading channels of $\kappa-\mu$ and $\eta-\mu$.

Yacoub, M.D. (2007)[14 ] dealt with two distributions of fading, namely the distribution channel of $\eta$ - $\mu$ fading and the distribution channel of fading $\kappa-\mu$. It comprised of Rice and Nakagami-m as unique cases, and a border was defined for the two fading distributions. This paper propounded that, $\eta-\mu$ fading model accounted for non-LOS communication conditions. Also, the $\kappa-\mu$ fading model seems to be adjustable in particular. Its notable utility and flexibility are exhibited clearly in this study. Thus we have considered the $\kappa-\mu$ fading model in our study for the FSO link of co-operative communication system under Dual-hop asymmetric DF relaying protocol.

\section{METHODOLOGY}

With context to co-operative wireless communication systems, Dual-hop relaying is highly adapted as spatial diversity presented by it. It can extend the coverage area efficiently under potentially severe condition of channel fading. RF connections are usually used in most relaying systems to establish complete end-to-end communication.

However, the scarceness of conventional dual-hop RF / RF communication which are licensed-spectrum resources along with the ever-increasing requirements of high-speed communication systems and need for more multi-user accessibility are some of the most critical constraints, FSO cooperative communication systems have incurred considerable research curiosity because of its enviable characteristics. As shown in Figure1 the proposed cooperative Relay Network Model consists of an " $\mathrm{S}$ " as the source node, an "R" as relay agent/node, and "D" as the destination/target node. Dual-hop Asymmetric FSO/RF systems are considered, where in the first hop, data shall be forwarded from the Source ' $S$ ' to the Relay ' $R$ ', and in other/next second hop, the data is released from the Relay ' $R$ ' to the Destination ' $D$,. For the Source to the Relay, the RF link is set up (S-R link), and the FSO link is used from the Relay Agent to the Destination (RD link).The S-R link follows Generalized-K fading distribution channel, while the link R-D follows $\kappa-\mu$ fading channel. The $\kappa-\mu$ fading distribution renders a general type multipath model for the Line of Sight (LOS) propagation scenario, which is then controlled by the two shaping parameters $\kappa$ and $\mu$ [21].
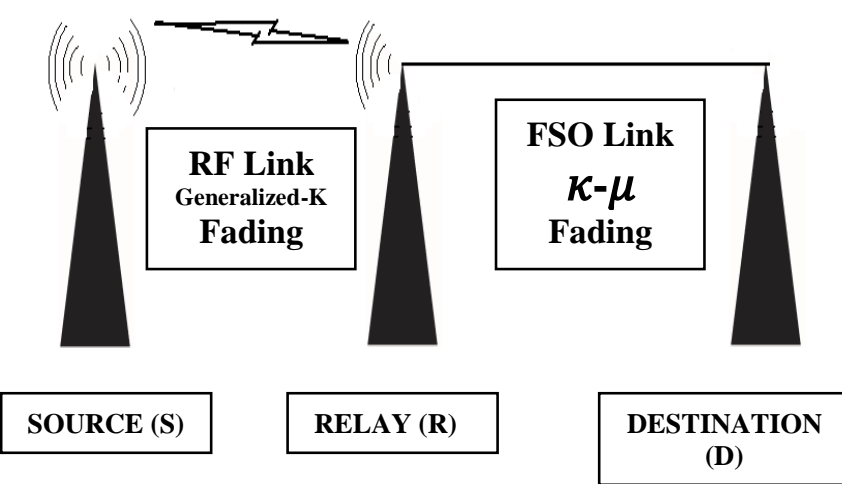

Figure 1: RF-FSO system Model

\section{A. RF (S-R) link}

The RF link follows Generalized- $\kappa$ fading distribution. The Generalized $-\kappa$ model combines Nakagami-m multipath fading in the S-R link. The PDF of Generalized $-\kappa$ fading channel in terms of instantaneous SNR can be expressed as given by [22] 
$p\left(\gamma_{s r}\right)=\frac{\alpha^{\beta+1}}{2^{\beta} \Gamma(m) \Gamma(\kappa)} \gamma_{s r}^{\frac{\beta-1}{2}} K_{\alpha}\left(a \sqrt{\gamma_{s r}}\right)$

Here $a=\sqrt{\frac{4 \kappa m}{\bar{\gamma}}}$ and other mentioned symbols in the formula the ' $\kappa$ ' and the ' $m$ ' are the shaping/molding parameters of Generalized- $\kappa$ combined with the Bessel function of order $\alpha$ is defined as the Nakagami-m channel distribution. Here $\alpha=\kappa-\mathrm{m}$, and $\beta=\kappa+\mathrm{m}-1$. Also $\mathrm{K}_{\alpha}(\bullet)$ exists as known Bessel function of order $\alpha$. Here $\alpha$ expression is in terms of $\bar{\gamma}$ which is the average value of the SNR.

\section{B. FSO (R-D) link}

The FSO communication systems have multiple merits such as inexpensive execution of installation, economical working costs because of license free spectrum, simple deployment, high data rate and immunity to interference. The FSO link of the proposed relay network follows $\kappa-\mu$ fading distribution. Rice that is Nakagami-n, Nakagami-m, one sided Gaussian, and Rayleigh distributions are unique cases of the proposed fading model [18]. The relation between the power of the main components and the wave power that are unevenly distributed is represented by $\kappa$, and $\mu$ is the non-negative natural real number. The PDF of $\kappa-\mu$ channel in $\mathrm{R}-\mathrm{D}$ link is expressed as

$p\left(\gamma_{r d}\right)=$

$\left\{I_{\mu-1}\left(2 \mu \sqrt{\kappa(1+\kappa) \Omega^{-1} \gamma_{r d}}\right)\right\} \times \frac{\mu(1+\kappa)^{\frac{\mu+1}{2}} \gamma_{r d}^{\frac{\mu-1}{2}}}{\kappa^{\frac{\mu-1}{2} e+\mu \kappa \Omega^{\frac{\mu+1}{2}}}} e^{-\frac{\mu(1+\kappa)}{\Omega}} \gamma_{r d}$

Where $\Omega=\mathbb{E}\left[\gamma_{r d}{ }^{2}\right]$, is the expectation operator. For unique cases, this $\kappa-\mu$ fading channel contains Rice $(\kappa=\mathrm{K}, \mu$ $=1)$, Nakagami-m $(\kappa=0, \mu=\mathrm{m})$ and one-sided Gaussian distribution $(\kappa=0, \mu=0.5)$.

\section{Assessment of capacity of Channel.}

Assessment of capacity of Channel is vital performance of wireless cooperative communication system. The extensive demand for wireless communication makes it imminent to ascertain the capacity limits of fading channels. The channel capacity serves as the higher limit of the data speed for reliable communication over noisy channel, and is defined in the paper in terms of outage probability. For deriving the expression of channel capacity, Outage probability formula is employed. And the formula for outage probability of $\kappa-\mu$ fading distribution is expressed in Equation (3)

$$
\begin{aligned}
P_{o u t}^{\kappa, \mu}= & \sum_{m=0}^{N}\left(\begin{array}{l}
N \\
m
\end{array}\right) Q_{\mu}^{m}(a, b) \times\left[1-Q_{\mu}(a, b)\right]^{N-m} \\
& \times\left[1-Q_{(m+1) \mu}(c, b)\right]
\end{aligned}
$$

Where $a=\sqrt{2 \mu \kappa} ; b=\sqrt{2 \mu(1+\kappa) \Omega^{-1} \Delta} ;$ $c=\sqrt{2(m+1) \mu \kappa}$

Also here $\mathrm{Q}$ is the generalized Marcum Q function that calculates the Marcum $Q$ of 2 non-negative real numbers ' $a$ ' and ' $b$ '. This generalized Marcum Q function is a built in tool function like in MATLAB, MATHEMATICA etc. and is expressed as follows:

$$
Q(a, b)=\int_{b}^{\infty} x \exp \left(-\frac{\left(x^{2}+a^{2}\right)}{2}\right) I_{0}(a x) d x
$$

Here $\mathrm{I}_{0}=$ modified Bessel function of the first kind with zero order.

Channel capacity for R-D link can be calculated using following formula for $\kappa-\mu$ fading channel distribution is given by

$$
C=\left(1-P_{\text {out }}^{\kappa, \mu}\right) B \log _{2}(1+\gamma)
$$

Therefore, the closed - form explicit channel capacity expression for the $\kappa-\mu$ fading channel distribution in FSO link and can be obtained by substituting Equation (3) in (5),

$$
C=\left(\begin{array}{c}
1-\sum_{m=0}^{N}\left(\begin{array}{l}
N \\
m
\end{array}\right) Q_{\mu}^{m}(a, b) \times\left[1-Q_{\mu}(a, b)\right]^{N-m} \\
\times\left[1-Q_{(m+1) \mu}(c, b)\right]
\end{array}\right) B \log _{2}(1+\gamma)
$$

\section{RESULTS AND DISCUSSION}

After procuring the expression for the channel capacity, Monte Carlo simulation method is used for verifying and validating the numerical expressions using MATLAB software. Channel capacity was plotted against SNR values for various $\kappa$ values ( $\kappa=1, \kappa=2, \kappa=3)$ as shown in the Figure 2. It is inferred from the graph that for higher $\kappa$ values, the channel capacity shows improved results.

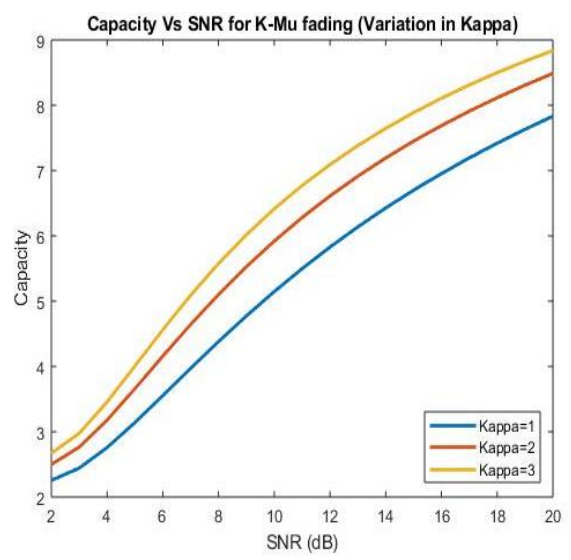

Figure 2: Channel capacity for different $\boldsymbol{\kappa}$ values Channel capacity was plotted against SNR values for various $\mu$ values $(\mu=1, \mu=2, \mu=3)$ as depicted in the Figure 3. It is deduced from the graph that, with the rise in the $\mu$ values, there is a significant improvement in the channel capacity.

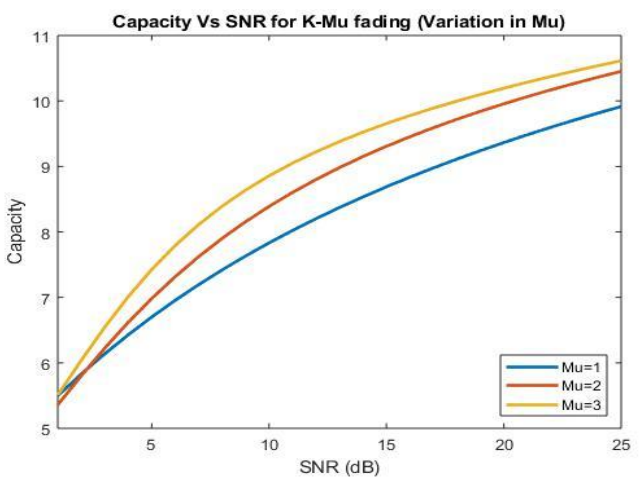

Figure 3: Channel capacity for different $\mu$ values

Published By:

Blue Eyes Intelligence Engineering \& Sciences Publication

(C) Copyright: All rights reserved.

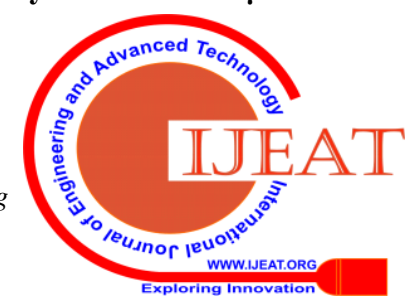




\section{Performance Assessment of Channel Capacity of Co-operative Communication based System over Dual-hop Asymmetric RF/FSO system}

\section{CONCLUSION}

The Channel capacity of co-operative relay system using DF relaying protocol is researched in $\kappa-\mu$ fading channel in the FSO link. Computationally efficient closed-form mathematical derivation for the capacity of noisy channel in terms of outage probability is delineated in the paper. Mathematical results have been drawn using MATLAB software to explain the enhancement in the capacity of the channel with respect to the escalation in the $\kappa$ and $\mu$ values of the $\kappa-\mu$ fading channel of R-D link of co-operative communication system. Using the findings obtained from this research, evaluation of the performance of channel communication can be undertaken in the future for the betterment of field of co-operative relaying communication system.

\section{REFERENCES}

1. Liu, K. R., Sadek, A. K., Su, W., \&Kwasinski, A. (2009). Cooperative communications and networking. Cambridge university press.

2. Nosratinia, A., Hunter, T. E., \&Hedayat, A. (2004). Cooperative communication in wireless networks. IEEE communications Magazine, 42(10), 74-80.

3. A. Sendonaris, E. Erkip, and B. Aazhang, "User cooperation diversity parts I and II," IEEE Trans. Commun., vol. 51, no. 11, pp. 1927-1948, Nov. 2003.

4. J. N. Laneman, D. N. C. Tse, and G.W.Wornell, "Cooperative diversity in wireless networks: efficient protocols and outage behavior," IEEE Trans. Inf. Theory, vol. 50, no. 12, pp. 3062-3080, Nov. 2004.

5. H. Nishiyama, M. Ito, and N. Kato, Relay-by-smartphone: realizing multihop device-to-device communications, IEEE Commun. Mag., vol. 52, no. 4, pp. 5665, Apr. 2014

6. W. Roh, J.-Y. Seol, J. Park, B. Lee, J. Lee, Y. Kim, J. Cho, K. Cheun, and F. Aryanfar, Millimeter-wave beamforming as an enabling technology for $5 \mathrm{G}$ cellular communications: theoretical feasibility and prototype results, IEEE Commun. Mag., vol. 52, no. 2, pp. 106113, Feb. 2014.

7. Palliyembil, V., Vellakudiyan, J., Muthuchidamdaranathan, P., \&Tsiftsis, T. A. (2018). Capacity and outage probability analysis of asymmetric dual-hop RF-FSO communication systems. IET Communications, 12(16), 1979-1983.

8. Chen, X., Lei, L., Zhang, H., \& Yuen, C. (2015). Large-scale MIMO relaying techniques for physical layer security: AF or DF?. IEEE Transactions on Wireless Communications, 14(9), 5135-5146.

9. Nauryzbayev, G., Rabie, K. M., Abdallah, M., \&Adebisi, B. (2018). On the Performance Analysis of WPT-Based Dual-Hop AF Relaying Networks in alpha-mu Fading. IEEE Access, 6, 37138-37149.

10. M. K. Simon and M.-S. Alouini, Digital Communication over Fading Channels, 2nd ed. New York: Wiley, 2005.

11. Tang, X., Wang, Z., Xu, Z., \&Ghassemlooy, Z. (2014). Multihop free-space optical communications over turbulence channels with pointing errors using heterodyne detection. Journal of Lightwave Technology, 32(15), 2597-2604.

12. Laourine, A., Alouini, M. S., Affes, S., \&Stéphenne, A. (2008). On the capacity of Generalized-K fading channels. IEEE Transactions on Wireless Communications, 7(7), 2441-2445.

13. Priyanka, C., Nithya, V., \&Bhaskar, V. (2018). k- $\mu$ fading channels: a finite state Markov modelling approach. Sädhanā, 43(1), 7.

14. D. B. da Costa and M. D. Yacoub, "Moment generating functions of generalized fading distributions and applications," IEEE Commun. Lett., vol. 12, no. 2, pp. 112-114, Feb. 2008.

15. N. Y. Ermolova, "Moment generating functions of the generalized $\eta-\mu$ and $\kappa-\mu$ distributions and their applications to performance evaluations of communication systems," IEEE Commun. Lett., vol. 12, no. 7, pp. 502-504, July 2008.

16. N. Y. Ermolova, "Useful integrals for performance evaluation of communication systems in generalised $\eta-\mu$ and $\kappa-\mu$ fading channels," IET Commun., vol. 3, no. 2, pp. 303-308, Feb. 2009.

17. K. Peppas, F. Lazarakis, A. Alexandridis and K. Dangakis "Error performance of digital modulation schemes with MRC diversity reception over $\eta-\mu$ fading channels," IEEE Trans. Wireless Commun., vol. 8, no. 10, pp. 4974-4980, Oct. 2009.
18. D. Dixit and P. R. Sahu "Performance of $L$-branch MRC receiver in $\eta \mu$ and $\kappa-\mu$ fading channels for QAM signals," IEEE Wireless Commun. Lett., vol. 1 no. 4, pp. 316-319, Aug. 2012.

19. M. D. Yacoub, "The $\kappa-\mu$ distribution and the $\eta-\mu$ distribution," IEEE Antennas. Propag. Mag., vol. 49, no. 1, pp. 68-81, Feb. 2007.

20. Miridakis, N. I., Matthaiou, M., \&Karagiannidis, G. K. (2014) Multiuser relaying over mixed RF/FSO links. IEEE Transactions on Communications, 62(5), 1634-1645.

21. Paris, J. F. (2014). Statistical Characterization of kappa-mu Shadowed Fading. IEEE Transactions on Vehicular Technology, 63(2), 518-526.

22. Laourine, A., Alouini, M. S., Affes, S., \&Stéphenne, A. (2008). On the capacity of Generalized-K fading channels. IEEE Transactions on Wireless Communications, 7(7), 2441-2445.

\section{AUTHORS PROFILE}

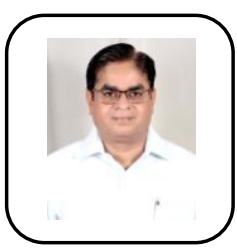

Shyam Baboo Bambiwal, received the B.E. degree in Electronics and telecommunications engineering from Shri Govindram Seksaria Institute of Technology and Science, Indore (M.P), India, affiliated to Devi Ahilya University, Indore (M.P.), India in 1993 and the M.E. degree in Electronics and communication Engineering from Delhi College of Engineering Delhi, India affiliated to Delhi University, Delhi, India, in 2008. Presently, he is working as Lecture (Electronics Engineering), and also In-Charge HOD of B.Voc (Mobile Communication) Department and In-Charge HOD of B.Voc (Software Development) department in Ambedkar Institute of Technology, (under DTTE Govt. of NCT of Delhi) Delhi -110092. He is pursuing his Ph.D. in Electronics and Communication from Dr. A.P.J. Abdul Kalam Technical University, Lucknow, India, formerly Utter Pradesh Technical University, and Mahamaya Technical University. His research interests include Electronics, communication and optical wireless communication

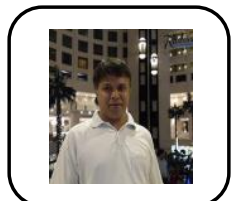

Dr. Rajveer Singh Yaduvanshi, received the Bachelor degree ( AMIE) in Electronics and communications engineering from IET, Kolkata, India in 1997 and the M.E. degree in Digital System in Electronics and communication Engineering from MNIT Allahabad, India, in 2000, also Ph.D in Electronics and communication Engineering from Delhi University, Delhi, India. Presently, he is working as Professor (Electronics and communication Engineering), in Ambedkar Institute of Advanced Communication Technologies and Research, (under DTTE Govt. of NCT of Delhi) Delhi 110031. His research interests include Electronics, communication, antennas and optical wireless communication.

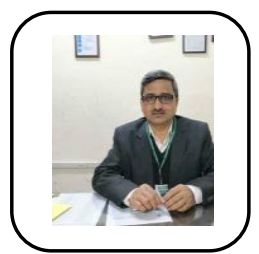

Dr. Vijay Kumar Pandey received the B.E in Electronics engineering from Bharati Vidyapeeth College of Engineering, Puna, affiliated to Poona University, Pune, Maharashtra 411043, India in 1990 and the M.E. degree in Control \& Instrumentation from Delhi College of Engineering, Delhi, affiliated to Delhi University, Delhi, India, in 1997, also Ph.D. in Electronics Engineering from Institute of Technology BHU, Varanasi, affiliated to Banaras Hindu University, Varanasi(U.P.), India in 2006. Presently, he is working as Professor (Electronics and communication Engineering) and Dean $\left(2^{\text {nd }}\right.$ Shift), in Department of Electronics and Communication Engineering in Noida Institute of Engineering and Technology, Knowledge Park-II, Institutional Area, Greater Noida, Uttar Pradesh 201306 India. His research interests include Electronics, communication, antennas and optical wireless communication.

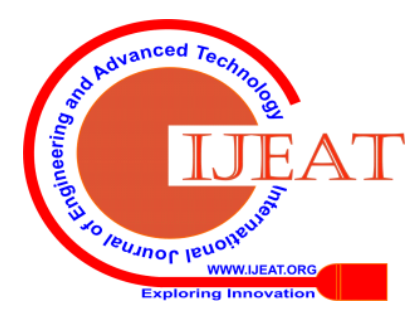

